\title{
Médiévales
}

Langues, Textes, Histoire

72 | printemps 2017

Roman du Genji et société aristocratique au Japon

\section{Un aspect de l'encyclopédisme de Thomas de Cantimpré. La section De lapidibus pretiosis du Liber de natura rerum}

An aspect of Thomas' of Cantimpré Encyclopaedism. The De lapidibus pretiosis Section of the Liber de natura rerum

\section{Mattia Cipriani}

\section{OpenEdition}

Journals

\section{Édition électronique}

URL : https://journals.openedition.org/medievales/8121

DOI : 10.4000/medievales.8121

ISSN : 1777-5892

\section{Éditeur}

Presses universitaires de Vincennes

\section{Édition imprimée}

Date de publication : 22 juin 2017

Pagination : 155-174

ISBN : 978-2-84292-612-0

ISSN : 0751-2708

\section{Référence électronique}

Mattia Cipriani, « Un aspect de l'encyclopédisme de Thomas de Cantimpré. La section De lapidibus pretiosis du Liber de natura rerum », Médiévales [En ligne], 72 I printemps 2017, mis en ligne le 28 février 2019, consulté le 23 avril 2022. URL : http://journals.openedition.org/medievales/8121 ; DOI : https:// doi.org/10.4000/medievales.8121 
Mattia Cipriani

\title{
Un aspect de l'encyclopédisme de Thomas de Cantimpré
}

\author{
La section De lapidibus pretiosis du Liber de natura rerum
}

Le Liber de natura rerum du Dominicain Thomas de Cantimpré $(1201-1270 / 1271)^{1}$ est une encyclopédie née ad usum Predicatorum pour une description de l'Univers propédeutique mais précise. L'œuvre a rencontré un grand succès et a connu une circulation fluide, même après la mort du religieux ${ }^{2}$ : toutefois, seules les rédactions les plus anciennes, qui sont conservées dans 129 manuscrits principalement de l'Europe du Nord et du Centre ${ }^{3}$, sont douées d'autorité : la première $(T h I)$ a été achevée

1. Les références et les citations renvoient à l'édition du Liber de natura rerum de Thomas de Cantimpré (désormais, $L N R$ et $\mathrm{TC}$ ) réalisée dans le cadre de notre thèse de doctorat (M. CIPRIANI, La Place de Thomas de Cantimpré dans l'encyclopédisme médiéval : les sources $d u$ Liber de natura rerum, Paris/Florence, 2014, vol. I-II ; dorénavant, MC I-II). Dans les notes n'est indiqué que le numéro des chapitres examinés; pour les titres, voir notre Annexe. On peut aussi se reporter à THOMAS CANTIMPRATENSIS, Liber de natura rerum, éd. H. Boese, Berlin/New York, 1973.

2. On en connait cinq versions avec de nombreuses variantes : sur cette question, voir B. K. VolLmanN, « La vitalità delle enciclopedie di scienza naturale », dans M. PICONE éd., L'enciclopedismo medievale, Ravenne, 1994,p. 135-146 ; E. FRUNZEANU, Les Configurations de la nature dans le Speculum maius de Vincent de Beauvais, Montréal, 2007, p. 39-40 ; B. VAN DEN ABEELE, «Diffusion et avatars d'une encyclopédie : le Liber de natura rerum de Thomas de Cantimpré », dans G. DE CALLATAY, B. VAN DEN ABEelE éd., Une lumière venue d'ailleurs, Turnhout, 2008, p. 141-176 ; M. SCHMITZ, Le Viridarium du juriste avignonnais Jean Raynaud, Louvain-la-Neuve, 2012, p. 94-180.

3. Désormais cités Th I et Th II. Pour une liste de ces témoins, voir B. VAN DEN AbEELE, «Diffusion... », p. 161-174, et MC I, p. XIII-XX, et MC II, p. 19-34 (qui propose quelques ajouts par rapport à la liste précédente). 
après $1241^{4}$, la seconde (Th II) vers $1256^{5}$ ). Elles ont en commun l'ordre Homme-Terre-Ciel des livres I à XIX et la disposition de la matière du général au particulier ${ }^{6}$; Th II ajoute pour sa part des passages, quelques chapitres supplémentaires et le livre XX. Bien que tout cela révèle d'emblée l'inclinaison pratique de l'auteur ${ }^{7}$, l'analyse de ses sources permet d'en approfondir la mens : en juxtaposant à la Bible des œuvres très hétérogènes (écrits « scientifiques », exégétiques, biographiques, chroniques, ou encore textes divertissants ${ }^{8}$ ), des notions de culture populaire et de courtes parties morales, il souligne en effet son intérêt pour le Créé tangible, qui éduque grâce à sa bonté intrinsèque. Le livre XIV, De lapidibus pretiosis, est un parfait exemple de sa démarche ${ }^{9}$.

4. Une hypothèse classique considère que Th I a été achevé entre 1237 et 1240 . La datation précise proposée ici dérive 1. de l'utilisation que fait Thomas de l'Anatomia de Richardus Anglicus (datée de 1242-1252), et 2. d'une allusion à la découverte, en Allemagne, d'une nouvelle mine d'étain $(\mathrm{XV}, 6)$ : cette dernière information est datée de 1241 par Matthieu Paris (Chronica maiora, éd. H. R. LUARD, 7 vol., Londres, 1872-1884, p. 151, et n. 4) ; la contemporanéité de cette découverte et du LNR est soulignée en outre par Thomas lui-même (modernis temporibus). Pour de plus amples références, voir Anatomia Ricardi Anglici, éd. R. TöPly EQUES, Vienne, 1902, p. V-VI ; G. W. CORNER, Anatomical Texts of the Earlier Middle Ages, Washington, 1927, p. 17 et 35-36 ; C. FERCKEL, Thomas von Cantimpré über die Metalle, dans J. RUSKA éd., Studien zur Geschichte der Chemie Festgabe, Berlin/ Heidelberg, 1927, p. 75-79 ; G. SARTON, Introduction to the History of Science, Baltimore, 1927-1948, vol. II, p. 657 ; K. SuDHOFF, « Der Micrologus : Text der Anatomia Richards des Engländers », Archiv fur Geschichte der Medizin, 19/3 (1927), p. 209-212.

5. Comme le prouvent les comparaisons avec le Bonum universale de apibus (1256) du même auteur (éd. G. ColvenERIUS, Douai, 1627, par exemple p. 100, 149, 245, 312, 333, 349, 540, 549), et le De mineralibus (1254-1261) d'Albert Le Grand (éd. A. BORGNET, Opera omnia, Paris, 1890-1899, vol. V, par exemple p.43). Sur ces dernières œuvres, voir D. WYCKOFF, Albertus Magnus Book of Minerals, Londres, 1967, p. XXXV-XLI ; B. FrIEDMAn, Albert the Great's Topoi of Direct Observation and his Debt to Thomas of Cantimpré, dans P. BINKLEY éd., Pre-Modern Encyclopaedic Texts, Leiden, 1997, p. 379392 ; P. CoRDEZ, « Materielle Metonymie. Thomas von Cantimpré und das erste Horn des Einhorns », Bildwelten des Wissens, Kunsthistorisches Jahrbuch für Bildkritik, 9 (2012), p. 85-92. Sur les rapports entre Thomas et Albert, on peut voir P. AIKEN, « The Animal History of Albertus Magnus and Thomas of Cantimpré », Speculum, 22 (1947), p. 205-225, et J. B. Friedman, Albert the Great's Topoi..., mais pas J. M. RidDle, J. A. Mulholland, «Albert on Stones and Minerals », dans J.A. WeISHEIPL éd., Albertus Magnus and the Sciences, Toronto, 1980 , p. 203-234. Pour des approfondissements, voir MC II, p. 25-28, 4546, 58, 183-188, 210-212, 233-236.

6. Dans l'ordre alphabétique ou dans l'ordre a capite ad calcem; les livres I, V et XIV contiennent aussi des chapitres monographiques sur l'obstétrique, la médecine vétérinaire ou la gravure, qui parachèvent l'aspect pratique de l'œuvre.

7. Sur 798 chap. de Th I, 765 concernent l'homme, les animaux, les végétaux et les minéraux ; et sur les 301 ajouts de $T h I I, 279$ portent sur ces sujets.

8. Autres exemples: Adam de Saint-Victor, Adélard de Bath, Aristote, Cophon, Jourdain de Saxe, Historia Brittonum, Recognitiones.

9. Abrégé en notes $\mathrm{Dlp}$. 


\section{Structure du De lapidibus pretiosis}

La section De lapidibus pretiosis se compose de 69 entrées organisées en trois blocs qui diffèrent par les sources, par le contenu et par la disposition de la matière ; Th II ajoute pour sa part De pyrophilo ${ }^{10}$.

Le premier chapitre, introductif, est augmenté dans Th II par un passage dialogique sur la formation des pierres. Les chapitres 2-69, rangés selon l'ordre alphabétique et de taille variable, décrivent les gemmes, indiquent leurs usages et pratiques, et indiquent si elles sont citées dans le livre de l'Exode ${ }^{11}$; Th II amplifie 21 de ces entrées par 22 insertions, deux leçons alternatives et le chapitre De pyrophilo. Le chapitre 70, monographique mais articulé, est divisé en segments techniques qui ne sont pas développés dans Th II ; les sections De sculphitos lapidum-Hucusque secundum antiquos et Item philosophum Tethes-De sculpturis, de 24 et 33 passages, décrivent les pouvoirs dérivés de l'art de la marqueterie, et sont introduites par des «doutes» qui reprennent le Prologus ${ }^{12}$ et sont probablement dus à Thomas: De sculphitos... se distingue ensuite de Item..., dans la mesure où il ne précise pas la pierre à graver ${ }^{13}$, et il se conclut par le bref Hucusque... dans lequel Thomas justifie son incrédulité par l'absence de commentaires aux textes-source ${ }^{14} ;$ Incipit...-Benedictio... se caractérise en revanche par le fait qu'il explique d'une manière pratique comment rendre aux pierres les pouvoirs qui ont été perdus avec le Péché originel.

\section{Sources et contenus}

Outre la structure du livre, le modus operandi de Thomas est visible aussi dans les œuvres et les contenus qu'il décide de copier : à l'instar des autres encyclopédistes du XIII ${ }^{\mathrm{e}}$ siècle, il invente en effet fort peu et se borne à choisir, retailler et arranger des auctoritates et des contenus selon ses propres objectifs.

Avant d'analyser les sources en question, il faut toutefois considérer l'état de la scientia lapidum du XIII ${ }^{\mathrm{e}}$ siècle. Les propriétés des pierres ont

10. Dlp 57.

11. Cf.Ex. 28, 31, 35, 36; et Marbodus RedonEnsis, Liber lapidum (éd. M. E. HERRERA, Paris, 2005, p. XXIII-XXIV).

12. Dlp 1: «opiniones antiquorum quas nec credendas per omnia credimus nec per omnia refutandas », «nec tamen per hoc credimus quod omnis sculptura virtutis signum et misterialis sit»; et $D l p 70$ : «nec approbande multum, nec penitus refutande [...]. Sed nec istis opinionibus in omnibus credendum existimo ».

13. Bien expliqué aussi dans K. MESLER, « The Medieval Lapidary of Tethel/Azareus on Engraved Stones and its Jewish Appropriations », Aleph, 14 (2014), p. 75-143.

14. Dlp $70:$ : in hoc magis dubitabiles sunt quia auctores earum minime invenimus annotatos ». 
toujours fasciné ${ }^{15}$, et l'Europe du Moyen Âge a vu naître plus de 200 œuvres sur ce thème. Par commodité, ces traités sont aujourd'hui regroupés en trois veines ${ }^{16}$; la première, pharmacologico-scientifique, explique les propriétés médico-magiques des gemmes en unissant les savoirs antiques, monastiques et populaires ; cette veine a rencontré un succès énorme ${ }^{17}$, et le livre XVI des Etymologiae d'Isidore de Séville († 636) ou le De lapidibus de Marbode de Rennes $(† 1123)$ en sont le parangon. Le second filon, magicoastrologique et d'origine orientale, décrit les pouvoirs dérivés d'intailles bien précises : il a eu une diffusion notable ${ }^{18}$ et comprend les Kyranides, traductions réalisées par Constantin l'Africain au XI ${ }^{\mathrm{e}}$ siècle (De physicis ligaturis de Costa ben Luca et De gradibus d'al-Jazzār), le De lapidibus de Damigeron-Evax et les Libri sigillorum de Tethel-Azareus ${ }^{19}$. Le filon

15. Sur cette question, voir M. STEINSCHNEIDER, « Lapidarien : ein culturgeschichtlicher Versuch », dans G. A. KoHUT éd., Semitic studies, Berlin, 1896, p. 42-72 ; M. Wellmann, «Die Stein- und Gemmenbücher der Antike», Quellen und Studien zur Geschichte der Naturwissenschaften und der Medizin, 4 (1935), p. 86-149; K. W. WIRBELAUER, Antike Lapidarien, Würzburg, 1937 ; C. BONNER, Studies in Magical Amulets, Chiefly GraecoEgyptian, Ann Arbor, 1950 ; A.CLOSS, Die Steinbücher in kulturhistorischer Überschau, Graz, 1958 ; A. Delatte, P. DeRChaIN, Les Intailles magiques gréco-égyptiennes, Paris, 1964 ; M. G. LANCELlOTTI, « Médecine et religion dans les gemmes magiques », Revue de l'histoire des religions, 218 (2001), p. 427-456.

16. Cf. J. Evans, Magical Jewels of the Middle Ages and the Renaissance, Oxford, 1922 ; L. THORNDIKE, A History of Magic and Experimental Science, New York, 1923-1958, vol. I, p. 494-497 ; G. SARTON, Introduction ..., vol. I, p. 764 ; J. M. RIDDLE, « Lithotherapy in Middle Ages : Lapidaries Considered as Medical Texts », Pharmacy in History, 12 (1970), p. 39-50 ; L. BIANCO, Le pietre mirabili, Palerme, 1992 ; N. WeILl-Parot, Les «Images astrologiques » au Moyen âge et à la Renaissance, Paris, 2002.

17. Cf. J. Evans, Magical Jewels..., p. 38 ; J. M. RiddLe, Marbode of Rennes (10351123), De lapidibus Considered as a Medical Treatise, Wiesbaden, 1977, p. XI-XII ; P. KITSON, « Lapidary Traditions in Anglo-Saxon England. Part I », Anglo-Saxon England, 7 (1978), p. 9-60 ; R. HAlleuX, J. Schamp, Les Lapidaires grecs, Paris, 2003, p. xvi ; L. BIANCO, Le pietre mirabili..., p. 20-22 ; C. GILIBERTO, «An Unpublished De lapidibus in its Manuscript Tradition », dans P. Lendinara, L. LAZZARI, M. A. D'ARONCO éd., Form and content of Instruction in Anglo-Saxon England in the Light of Contemporary Manuscript Evidence, Turnhout, p. 251-283 ; ID., «La letteratura mineralogica nel mondo germanico medievale, con particolare riguardo per il lapidario antico inglese et il lapidario di Prüll », dans L. Vezzosi éd., La letteratura tecnico-scientifica nel Medioevo germanico, Alessandria, 2009, p. 95-118.

18. $C f$. D. PINGREe, "The Diffusion of Arabic Magical Texts in Western», dans B. SCARCIA AMORETti éd., La diffusione delle scienze islamiche nel Medio Evo europeo, Rome, 1987, p. 57-102 ; R. HALLEUX, J. SCHAMP, Les Lapidaires grecs..., p. 219-221, 230234 ; M. L. LANCELLOTTI, « Médecine et religion dans les gemmes magiques »....; P. JONES, «Amulets and Charms », dans M. RuBIN éd., Medieval Christianity in Practice, Princeton, 2009, p. 194-199 ; K. MESLER, « The Medieval Lapidary of Tethel/Azareus... », p. 75-106.

19. $C f$. M. SteINSCHNEIDER, «Übersetzer aus dem Arabischen: ein Beitrag zur Bücherkunde des Mittelalters », Serapeum, 31 (1870), p. 289-298, 305-331. Sur le lapidaire dit de Theel (ou Thetel ou Tethel) et l'opuscule d'Azareus, on verra plus récemment N. WeILLPAROT, Les «Images astrologiques »..., notamment p. 113-122. (NdT) 
biblico-symbolique, pour sa part, explique la minéralogie d'Aaron (Ex., 28.17-20), du roi de Tyr (Ez., 28.13), et de la Jérusalem céleste (Ap., 21.19$20)^{20}$ : diffusé surtout au début du Moyen Âge, il inclut la traduction partielle de l'œuvre grecque d'Épiphane de Salamine (ou de Chypre, IV ${ }^{\mathrm{e}}$ siècle) et les lapidaires de saint Augustin (De doctrina christiana, L. III.16, et De Genesi contra Manicheos, L. II.10), Bède (Explanatio Apocalypsis), Hraban Maur (De universo, L. XVII), et Hildebert de Lavardin († 1133), évêque du Mans.

Malgré une telle variété, Thomas de Cantimpré ignore les « classiques » minéralogiques et christiano-symboliques ${ }^{21}$; il considère avec réserve la minéralogie astrologique, fait se côtoyer des traités médicomagiques avec des textes naturalistes hétérogènes, et ne reprend directement ni les lapidaires de Galien, Dioscoride, Oribase, Aristote, ou Thebit ${ }^{22}$, ni

20. $C f$. D. Pingree, «The Diffusion of Arabic Magical Texts...», p.64-67; C. GILIBERTO, « An Unpublished...», p. 254-255, n. 15-19.

21. Consultés en édition: EPIPHANIUS, De XII gemmis rationalis (éd. O. GuENTHER, Prague/Vienne/Leipzig, 1898, p. 743-773) ; RABANUS MAURus, De universo (éd. J.-P. MigNE, Paris, 1844-1865, PL, vol.91, col. 462-474) ; BEDA, Explanatio Apocalypsis (éd. P. KITSON, «Lapidary... Part II », Anglo-Saxon England, 12 (1983), p. 73-123) ; HiLdEBERTUS, Carmina minora (éd. A. B. ScotT, Leipzig, 1969) ; cf. P. Kitson, « Lapidary... Part I... », p. 20-22.

22. Pseudo-Galenus, De simplicibus medicamentis ad Paternianum (Omnia Cl. Galeni Pergameni, Basilae, 1542, vol. IV, t. VIII, col.299-346); DioscorIDES, Dioscorides Longobardus (éd. H. STADLER, «Dioscorides Longobardus (Cod. Lat. Monacensis 337), liber V », Romanische Forschungen, 13 (1902), p. 161-243), et Dioscorides Alphabeticus (ms. Coligny, Fondation Martin Bodmer, Cod. Bodmer 58, f. 1r-78v: <http://www.ecodices.unifr.ch/it/list/one/fmb/cb-0058>); MACER FLORIDUS, De viribus herbarum (éd. L. Choulant, Leipzig, 1832) ; Oribasius, Synopsis (éd. A. Molinier, «Anciennes traductions latines de la Synopsis et des Euporistes », dans U. C. BUSSEMAKER, C. DAREMBERG éd., Euvres d'Oribase, Paris, 1876, vol.VI) et ID., Euporistes (éd. A. Molinier, «Anciennes...», vol. VI); ARISTOTELES, De lapidibus (éd. V. Rose, «Aristoteles De lapidibus und Arnoldus Saxo », Zeitschrift für deutsches Altertum und deutsche Literatur, 18 (1875), p. 349-397, et éd. J. RuSKa Das Steinbuch des Aristoteles, Heidelberg, 1912, p. 183-208) ; THEBIT BENCORAH, De imaginibus (éd. F. J. CARMODY, Astronomical Works, Berkeley, 1960, p. 180-198) ; CONSTANTINUS, De incantationibus et adiurationibus epistula et De gradibus (CONSTANTINI AFRICANI Opera, Basileae, 1536, p. 317-320 et 342-387) ; Damigeron-EvaX, De lapidibus (éd. R. HalleuX, J.SChamp, Les Lapidaires grecs..., p. 229-297 ; éd. J. Evans, op. cit., p. 195-216) ; Kyranides (éd. L. DelatTe, Paris, 1942) ; Avicenna, Liber Canonis, Venetiis, 1505 ; IoHANnes SERAPIOn, Practica, Lugduni, 1525, f. 1r-168r ; RHAZES, Liber sexaginta animalium (RASIS Opera, Venetiis, 1500, f. 104v-109v). $C f$. V. Rose, «Aristoteles De lapidibus...», J. RusKA, Das Steinbuch des Aristoteles...; C. F. SEYBOLD, «Rezension zu Julius Ruska, Das Steinbuch des Aristoteles », Zeitschrift der deutschen Morgenländischen Gesellschaft, 68 (1914), p. 606-625 ; M. WELLMANN, «Aristoteles De lapidibus », Sitzungsberichte der preussischen Akademie der Wissenschaften, (1924), p. 79-82 ; F. KLEIN-FrANKE, « The Knowledge of Aristotle's Lapidary during the Latin Middle Ages», Ambix, 17 (1970), p. 137-142 ; J. M. RIDDLE, «Lithotherapy...», p. 44-45 ; ID., Marbode of Rennes..., p. 1-14 ; J. M. RidDLE-J. A. MULHOLland, Albert on Stones and Minerals...; D. PIngREe, "The Diffusion of Arabic Magical Texts... », p. 6668 ; R. HAlleuX, J. SCHAMP, Les Lapidaires grecs..., p. 211-212. Sur les rapports entre Kyranides et Liber de natura rerum, cf. MC II, p. 22-25, 149-150. 
les traductions de Constantin ou les parties lithologiques des Kyranides, de Damigeron, d'Avicenne, de Serapion l'Ancien ou de Rhazès ${ }^{23}$. Le Brabançon n'emploie pas davantage les De lapidibus symboliques et - même si l'on ne peut exclure l'emploi d'une liste des pierres d'Aaron ${ }^{24}$ il se reporte directement à la Bible et à la Glose pour les gemmes du livre de l'Exode ${ }^{25}$.

Dans la section De lapidibus pretiosis, Thomas de Cantimpré recopie donc : le De lapidibus de Marbode avec les gloses de DamigeronEvax, les Etymologiae d'Isidore, le Liber Tethel-Azareus complété par des bénédictions, les Metereologica, le De civitate Dei, l'Historia scholastica, l'Exode et la glose, un liber en vers, la Naturalis historia de Pline, le Polyhistor de Solin, l'Hexaëmeron de Bède ${ }^{26}$, le De aromaticis speciebus $\mathrm{du}$ Pseudo-Platearius ${ }^{27}$, et des textes médicaux inconnus à orientation pratique et descriptive; on trouve en outre de rares similia avec Jacques

23. On trouve des loca similia avec certaines de ces œuvres dans Dlp 12 (Avicenne) ; 31 (Sérapion) ; 58 (Rhazès).

24. Souvent en marge d'œuvres majeures : voir J. Evans, M. S. SERJEANTSOn, English Mediaeval Lapidaries, Londres, 1933, p. 16-37, 147-157 ; C. GILIBERTO, « La letteratura...», p. 98 ; et infra.

25. Sur les sources lithologiques de l'encyclopédisme, $c f$. J. M. RIDDLE, «Lithotherapy...» ; ID., Marbode of Rennes..., p. 11-14 ; J. M. RIDDLE, J. A. MulHolland, Albert on Stones and Minerals..., p. 232-234; R. HalleuX, «Damigeron, Evax et Marbode. L'héritage alexandrin dans les lapidaires médiévaux », Studi Medievali, 1 (1974), p. 327-347; R. HAlleuX, J. Schamp, Les Lapidaires grecs..., p. 208-214 ; C. LeCouteuX, « Arnoldus Saxo : Unveröffentlichte Texte, transkribiert und kommentiert», Euphorion, 76 (1982), p. 389-400 ; ID., Dictionnaire des pierres magiques et médicinales, Paris, 2011 ; D. PINGREE, «The Diffusion of Arabic Magical Texts... », p. 66-69 ; L. STURLESE, « Florilegi filosofici e enciclopedie in Germania nella prima metà del Duecento », Giornale critico della filosofia italiana, 69 (1990), p. 295-319 ; I. DRAELANTS, « Introduction à l'étude d'Arnoldus Saxo et aux sources du Defloribus rerum naturalium », dans C. MEIER, S. SCHULER, M. HECKENKAMP éd., Die Enzyklopädie im Wandel vom Hochmittelalter bis zur Frühen Neuzeit, Munich, 2002, p. 85-122 ; EAD., «La science encyclopédique des pierres au $13^{\mathrm{e}}$ siècle : l'apogée d'une veine minéralogique », dans C. THOMASSET, J. DuCOS, J.-P. CHAMBON éd., Aux origines de la géologie de l'Antiquité au Moyen Âge, Paris, 2010, p. 91-139.

26. Marbodus Redonensis, Liber lapidum..., et éd. J.M. Riddle, Marbode of Rennes..., p.34-92; DAMIGERON-EvaX, De lapidibus...; IsIDORUS HISPALENSIS, Etymologiae (éd. W. M. LindSAY, Oxford, 1911, vol. II) ; ARISTOTELES, Metereologica (éd. P. L. Schoonheim, Leiden/Boston/Cologne, 2000); Augustinus, De civitate Dei (éd. B. Dombart, A. KALB, Leipzig, 1981) ; Petri COMESTORIS Historia scholastica, Lugduni, 1542 ; Biblia Latina, Basileae, 1498-1502 ; PHILIPPE DE THAON, Lapidaire (éd. J. EVANS, P. STUDER, Anglo-Norman Lapidaries, Paris, 1924, p. 200-259, et éd. P. MEYER, «Les plus anciens lapidaires français», Romania, 38 (1909), p. 496-552) et PHILIPPE DE Thaon, Bestiaire (éd. E. Walberg, Paris, 1900) ; Caius Iulius Solinus, Polyhistor (éd. Th. Mommsen, Berlin, 1894) ; Gaius Plinius Secundus, Naturalis Historia (éd. L. IAN, C. Mayhoff, Leipzig, 1892-1909); BedA, Hexaëmeron (éd. J.-P. Migne, PL, vol.91, col. 9-190).

27. Non identifié : malgré l'homonymie, il ne s'agit pas du Circa instans de LNR X, XII et XII, analysé dans MC II, p. 179-183. 
de Vitry, Alexandre Neckam et Hildegarde de Bingen ${ }^{28}$. En revanche, le religieux étoffe Th II avec d'autres données tirées de Marbode, de Pline et de textes inconnus (un ensemble de questiones et responsiones ${ }^{29}$, un lapidaire médical, un texte à orientation pratique et descriptive, la Scriptura Esculapii ad Octavianum avec un liber de serpentibus $)^{30}$.

Ces choix rendent manifeste l'inclination médico-naturaliste de Thomas de Cantimpré, et cette tendance est encore soulignée par les contenus qu'il choisit: il sélectionne en effet des notices en fonction de l'aspect, de la localisation, et des capacités intrinsèques (thérapeutiques ${ }^{31}$, « personnelles », sociales) ${ }^{32}$ des gemmes, en évitant de renvoyer à la magie, aux intailles et aux croyances particulières ou antiques ; semblables omissions - cohérentes avec le choix d'auctoritates «concrètes » comme Marbode et Isidore - sont visibles en particulier dans les chapitres 2-70, où le Brabançon ne reprend pas - ou laisse de côté - 28 passages à caractère mythologique ou magique sur les Muses, Milon de Crotone, Vénus, Zoroastre, Metrodore, différents monstres et fantômes, Jupiter, Castor et Pollux, les Perses, Théophraste, Deendor, Circé, les Mèdes, Syrte, la Lybie, l'hydromancie, les Scythes, la Bactriane, le Nil, Néron, les gladiateurs, la divination, Phaëton, Elector, le fleuve Eridan, la Perse, et remplace les noms de planètes et de divinités antiques (Jupiter, Vénus...) par des termes aussi vagues que homo/femina ${ }^{33}$.

Cette propension du Dominicain est encore plus nette si l'on considère que, bien qu'il achève son chapitre avec une bénédiction ${ }^{34}$ et répète à maintes reprises que les pouvoirs des gemmes dérivent de $\mathrm{Dieu}^{35}$, il n'y a que dans le chapitre sur le béryl, De berillo, qu'il insère un passage

28. Entre Dlp 4,28,44/Orientalis historia 91.194, 196-197, et 88.180 (éd. J. DoNNADIEU, Turnhout, 2008, p. 378, 380 et 356) ; Dlp 1, 12/De naturis rerum II, 94, et De laudibus divinae Sapientiae VI, v. 229-230 (éd. Th. WRIGHT, Londres, 1863, p. 180 et 468), et Dlp 70/Liber subtilitatum, IV, Praefatio.1 et 5-6 (éd. J.-P. MiGnE, PL, vol. 197, col. 1247-1250).

29. Sur ces œuvres dans $L N R$, voir I. DRAELANTS, « La question ou le débat scolastique comme formes du discours scientifique dans les encyclopédies naturelles du XIII ${ }^{\mathrm{e}}$ siècle : Thomas de Cantimpré et Vincent de Beauvais », Scientiarum historia, 31 (2005), en particulier p. 128-129 ; et MC II, p. 37, 56-57, 124-127, 207-209.

30. Voir notre Annexe.

31. Déjà dans $D l p 1$ : «Quippe magna virtus eorum videtur et efficacia sanitatum », et, comme on vient de le dire, plus développé dans Th II.

32. Sur cette question, voir F. D. ADAMS, The Birth and Development of the Geological Sciences, Baltimore, 1938, p. 137-169 ; E. DI VENOSA, « Diffusione e ricezione dei trattati mineralogici in area tedesca », dans F. FERRARI, M. BAMPI éd., Le lingue e le letterature germaniche fra il XII et il XVI secolo, Trente, 2002, p. 351-365.

33. Dlp $3 ; 7 ; 15 ; 26 ; 28 ; 44 ; 46 ; 58 ; 59 ; 65 ; 67 ; 70$.

34. Dlp 70 ; il est intéressant de noter que, malgré son incrédulité, Thomas fait une place à ces sections.

35. Dlp $1 ; 22 ; 35 ; 70$. Cf. C. BuRnETT, «Adelard, Ergaphalau and the Science of the Stars », dans ID. éd., Adelard of Bath, Londres, 1987, p. 133-145 ; N. WEILL-PAROT, Les «Images astrologiques »..., p. 119-122. 
symbolico-moralisant sur les dons de Marie $^{36}$; cette section est de toute façon très particulière car c'est la seule, de tout le De lapidibus pretiosis, qui soit versifiée.

Enfin, son approche est soulignée par plusieurs excerpta bibliques, ajouts et similia eux aussi exclusivement descriptifs, bien que tirés de sources qui ne sont pas à proprement parler naturalistes, comme le De civitate Dei, l'Historia scholastica, la Bible et la Glose.

\section{Organisation et style}

Le modus pensandi de Thomas de Cantimpré est également révélé par la précision avec laquelle il utilise et agence ses sources. Il construit ses chapitres en copiant avec soin les « colonnes portantes ${ }^{37}$ et en les unissant en phrases et paragraphes cohérents, dont l'ordre est souvent différent de celui de l'original (par exemple, source $A^{\text {portion } 1}-B^{2}-A^{4}-A^{2}-B^{l}$ ). Ces noyaux de base sont interpolés par des noms, des notions, des affirmations tirées de sources secondaires, qui réélaborent la matière d'origine et la complètent avec des éléments descriptifs ou spécialisés (A source $A^{5}-A^{3}-c$ ). Quand les informations sont maigres, le frère n'hésite de toute façon pas à créer un lemme à partir d'une auctoritas unique ou de sources complémentaires ; ces entrées sont souvent développées dans $T h I^{38}$.

Le De lapidibus, les Etymologiae et le De sigillis sont ainsi les textes de base du De lapidibus pretiosis : ces œuvres sont de fait copiées in extenso et avec précision dans 62 de ses 70 chapitres $^{39}$. Le rôle principal revient à Marbode, qui ouvre, développe et conclut avec des éléments médicopratiques la plus grande partie des chapitres specialiter; ce choix tient probablement à la cohésion, la complétude et la simplicité de cette source. Quant aux Etymologiae, elles complètent le De lapidibus pretiosis avec des

36. Dlp 11. De fait, ce livre manque de renvois à des sources symboliques importantes pour d'autres sections du LNR, à savoir la Bible et le Physiologus (vu dans les versions : $A$ [ms. Bruxelles, BR, 10066-77, f. 140r-156v] ; $B$ [éd. F. J. Carmody, Paris, 1939] ; $B$ Is [Guillaume le Clerc, Bestiaire, éd. M. F. Mann, Heilbronn, 1888, p. 37-73] ; $Y$ [éd. F. J. CARMOdY, «Physiologus latinus : versio Y», Classical Philology, 12 (1941), p. 95134] ; ont été également pris en compte F. SBordone, Physiologus, Milan-Rome, 1936, et TheOBALDI EPISCOPI Physiologus de naturis duodecim animalium (éd. P. T. EDEN, Leyde, 1972).

37. Ces sources changent selon le sujet et les intérêts : pour les régions de l'air (L. XVI), on trouve par exemple Michel Scot, et pour les éléments et le ciel (L. XIX-XX), Guillaume de Conches.

38. Sont dérivés de sources secondaires (Exode, Glose, Isidore, Bède, pseudo-Platearius) les passages suivants : Dlp 50 ; 57 ; de Marbode : Dlp $6 ; 7$; 8 ; $14 ; 16 ; 18 ; 19 ; 20 ; 26$; 27 ; $34 ; 35 ; 43 ; 45 ; 48 ; 49 ; 52 ; 53 ; 54 ; 55$; 60 ; 68 ; d'Isidore : Dlp 39 ; $40 ; 47,63$; 66 ; de Solin : Dlp 24. Font l'objet d'un plus ample développement : Dlp 8; 14 ; 16 ; 18 ; 19 ; 20 ; $27 ; 40 ; 52 ; 54 ; 66$.

39. Voir notre Annexe et I. DraELANTS, « La science... ». 
éléments, comme l'aspect, l'origine ou les particularités des pierres absentes chez Marbode ( 9 chapitres $^{40}$ ) ; il est fort possible aussi que les Etymologiae aient été retenues à cause du prestige dont jouissait Isidore de Séville au $\mathrm{XIII}^{\mathrm{e}}$ siècle $^{41}$. Enfin, bien que son emploi soit limité au chapitre 70 , le $D e$ sigillis est la seconde source du De lapidibus pretiosis (pour 58 passages à dominante pratique ou descriptive), et son usage marque une des premières tentatives de Thomas pour augmenter le caractère pragmatique de son Liber de natura rerum ${ }^{42}$.

Avant de poursuivre, il est utile d'observer la façon dont Thomas se comporte avec Marbode. De fait, il en sélectionne et en paraphrase les vers en fonction de ses propres objectifs; outre qu'il modifie l'ordre de l'auctoritas, un tel usage accentue des erreurs typiques de Thomas, à savoir la fusion, la duplication et la réinterprétation de données semblables ${ }^{43}$. Dans 9 chapitres $^{44}$, Thomas mêle à Marbode des éléments descriptifs ou thérapeutico-personnels tirés de Damigeron-Evax : considérant leur rareté, leur brièveté, leur anonymat et leur état corrompu, il semble puiser ces parties directement dans le manuscrit de Marbode qu'il utilisait ; impression renforcée tant par l'analyse du modus scribendi du Brabançon - qui tend à incorporer, sans le dire, des gloses à ses manuscrits-sources ${ }^{45}-$, que par l'examen de la tradition complexe du De lapidibus, souvent contaminée,

40. Dlp $39 ; 40 ; 47 ; 62 ; 63 ; 66$; avec Pline pour Dlp $6 ; 64 ; 65$.

41. $C f$. I. DraElanTS, «Encyclopédies et lapidaires médiévaux: la durable autorité d'Isidore de Séville et de ses Étymologies », dans J. ElFASSI, B. RiBÉMONT, N. MARGOLIS éd., La Réception d'Isidore de Séville durant le Moyen Âge tardif (XII ${ }^{e}-X V^{e}$ siècle), Orléans, 2008, p. 39-93.

42. Voir notre Annexe.

43. Traits communs à toutes les sections alphabétiques du Liber de natura rerum, comme on peut le voir par exemple dans les chap. IV, 14 (dans lequel Thomas, comprenant mal Pline, donne au castor les caractéristiques du veau de mer), 80-81 (où il redouble les onagres) et 9 (dans lequel il «crée » l'animal ana à partir du poisson amia cité par Aristote). Dans $D e$ lapidibus pretiosis, aux chap. $9 ; 20 ; 40 ; 50$ (et déjà dans $L N R$ XI, 27) ; $52 ; 55 ; 57$ (dans Th II) ; 60 ; 67 ; pour approfondir la question, comparer Dlp avec Marbode, De chrisolitochriselectro (p. 49, 175) ; De optallio (p. 153); De sardonice-onice-sardio (p. 43, 45, 47) ; De silentite-chelonite (p. 93, 131) ; et Isidore, cap. XVI, 14.6 ; 4.18-4.19; 8. Sur la démarche de Thomas, voir MC II, p. 45-47, 51-53.

44. Dlp 4 (avec Damigeron-Evax, versio BAM, p. 238-239) ; 11 (versio CPT, p. 276) ; 15 (versio BAM, p. 246) ; 17 (ibid., p. 243) ; 32 (ibid., p. 259); 37 (dans Th II, con ibid., p. 292) ; 44 (ibid., p. 259) ; 58 (ibid., p. 261-262) ; 69 (ibid., p. 267-268). La corruption du Damigeron-Evax de Thomas est visible en $D l p 58$, où les passages originaux « si fieri potest tangat capita vinculi statimque rumpentur» et «Idem nascitur et in Apulie regione apud Lucaniam » deviennent : «fugat etiam morbum illum horribilem qui vulgariter dicitur nolime-tangere, sed colore post fugationem dicitur vitiari » et «In Ypoio alumnie lapides saphiri sunt ». Le fait que Thomas rapporte des passages quasiment indéchiffrables montre bien qu'il s'intéresse plus à la transmission des données qu'au résultat.

45. Autres exemples dans le Liber de natura rerum, IV, 50 VII, 1, où Thomas recourt à des marginalia à l'Historia scholastica comme s'il s'agissait du corps du texte ; voir Historia scholastica, Lugduni, 1542, respectivement f.103r et 5v). Sur cette question, 
même si c'est à la marge, par des versions alphabétiques corrompues de Damigeron-Evax ${ }^{46}$.

Viennent en revanche compléter le De lapidibus pretiosis des œuvres rarement copiées (Meteorologica, Historia scholastica, De aromaticis speciebus, Hexaëmeron, les vers sur le béryl, De berillo), des textes employés plus fréquemment mais, dans ce chapitre, de manière limitée (la Naturalis historia, le Polyhistor, la Bible, la Glose, des ouvrages médicaux, pratiques, descriptifs ${ }^{47}$ ), sources de $T h I I$ : c'est de ces dernières en effet que proviennent seulement les brefs excerpta dialogiques, médico-personnels, technico-naturalistes et descriptifs ${ }^{48}$ qui renforcent la dimension pratique du Liber de natura rerum, mais qui sont marginaux dans l'économie du De lapidibus pretiosis.

En outre, même les rares interventions du frère sont elles aussi accessoires : ces brèves interpolations - qui, mise à part l'expression de doutes, sont les seules actions manifestes de Thomas - n'altèrent pas les sources, et consistent en commentaires attribuant des bizarreries aux pierres d'Orient ${ }^{49}$, ou en ajouts ${ }^{50}$ et similia venant étoffer des données un peu maigres. Parmi les sources secondaires, enfin, Augustin fait l'objet d'un traitement particulier : bien qu'il soit marginal dans le livre XIV, il en est bel et bien une pierre angulaire du point de vue idéologique ${ }^{51}$; et cette position est typique de Thomas, qui fonde le Liber de natura rerum sur des idées fortement aristotéliciennes et augustiniennes.

voir E. FRUNZEANU, E. KUHRY, «L'apport des gloses, des paraphrases et des syntagmes synonymiques à la compréhension des textes », Spica, 1, 2011, p. 39-49.

46. Sur cette tradition complexe, voir M. E. HERRERA, « La historia del texto del Liber lapidum de Marbodo de Rennes a través de los manuscritos provenientes de las bibliotecas francesas del siglo XII », dans D. NeBBIAI-DAlla GuARDiA, J.-F. GENEST éd., Du copiste au collectionneur, Turnhout, 1998, p. 153-168; EAD., Liber..., p. XLVI-XLVII, et aussi P. KITSON, « Lapidary... Part I... », p. 19 ; R. HALlEUX, J. SCHAMP, Les Lapidaires grecs..., p. 226-228 ; I. DraELANTS, «La science... », p. 111. Pour des exemples de contaminations similaires, voir le ms. Paris, BnF, lat. 8454 dont il est question infra.

47. Dlp $2 ; 11 ; 12 ; 13 ; 22 ; 31 ; 38 ; 41 ; 50 ; 58 ; 65$.

48. Dans Dlp 1 ; 41 (introduit par questio quomodo fiunt, queritur autem quomodo fiat, respondemus, solvimus). Dlp $8 ; 9 ; 14 ; 15 ; 16 ; 19 ; 20 ; 24 ; 27 ; 30 ; 33 ; 37 ; 40 ; 54 ; 60$ (à partir de mitigat ou valet). Dlp $37 ; 57$. Dlp $41 ; 51 ; 52 ; 66$.

49. Dlp 1 (dans $T h I) ; 25 ; 34 ; 44$ (introduits par lapis est Orientis).

50. Dlp $4 ; 9 ; 15 ; 21 ; 22 ; 42 ; 46 ; 50 ; 50 ; 51$ (à 1'exception d'une allusion dans le Liber lapidum [éd. P. MEYER, «Les plus anciens lapidaires français »..., p. 551], ces pouvoirs ne sont présentés que par Thomas de Cantimpré : ce qui n'exclut pas une mauvaise compréhension de Marbode) ; 55 ; 59 ; 60 ; 61 ; 62 ; 67 ; 68 (de secundum quosdam, lapis est pretiosissimus ou ut ipse dicit).

51. Dlp 1: «in hoc sequimur gloriosissimum Augustinum». Rappelons que Thomas fut ermite de saint Augustin (à partir de 1217) avant de devenir Dominicain en 1231 : ce qui peut justifier sa fidélité envers l'évêque d'Hippone, en particulier à son De doctrina christiana. Voir à ce sujet MC II, p. 17-18, 74-79 et 243-244 (et p. 15 pour des références bibliographiques). 
Pour conclure, Thomas ne donne dans son De lapidibus pretiosis que peu de pistes pour comprendre quand il change l'identité des auctoritates concernées par son style « au croisement $»^{52}$ : ce qui rend complexe leur identification, mais souligne aussi la préparation du frère, qui devait avoir une culture fort étendue pour « sauter » ainsi au milieu d'œuvres variées ${ }^{53}$.

\section{Méthode et bibliothèque}

L'étude du De lapidibus pretiosis permet, pour finir, de considérer la question des stratégies et de la bibliothèque de Thomas. Si l'on tient compte surtout de son modus scribendi, il semble que le frère ait eu trois manières de travailler. L'ampleur de son texte et la fidélité de sa copie font penser qu'il a reproduit des sources minéralogiques surtout de première main, et non à travers des abréviations ou compilations ${ }^{54}$; le De lapidibus de Marbode en est un bon exemple: Thomas passe en effet de l'un à l'autre de 54 des 60 chapitres qui le composent, recopiant 450 de ses 732 vers $^{55}$. De nombreuses erreurs du Brabançon semblent en revanche découler d'une distance par rapport à sa source : de fait, il semble reproduire à certains moments les auctoritates d'après des textes intermédiaires. Le caractère générique des similia fait penser aussi que dans un cas ou deux au moins, le religieux a introduit des connaissances personnelles et des rappels (mnémotechniques, de troisième main...) dérivant du milieu culturel de son temps.

En ce qui concerne les manuscrits dont disposait notre auteur, il faut donner toute sa valeur au De sigillis, un lapidaire magico-astrologique mêlant l'ouvrage de Thetel sur les pierres précieuses ainsi que l'opuscule dit d'Azareus. Cette famille de textes combine, dilate et restructure toujours de manière différente des noyaux constants d'éléments ${ }^{56}:$ chacun des textes

52. C'est le cas avec 7 excerpta d'Isidore (Dlp $5 ; 39 ; 40 ; 46 ; 63 ; 65 ; 66), 3$ de la Glossa super Exodum (Dlp $50 ; 61), 2$ de Pline et Solin (Dlp $44 ; 69$; et 19; 45, où il renvoie à $L N R$ VII), et 1 des Metereologica, d'Augustin (tous deux Dlp 1), de Bède, de Platearius (tous deux Dlp 50), de la Scriptura Esculapii, libro de serpentibus (tous deux Dlp 57), de Tethel (Dlp 70); ces passages sont introduits par ut dicit, sicut scribit, auctor libri, in libro, secundum librum. Rappelons que le Dominicain, dans son Prologus, récapitule les auctoritates employées dans son De lapidibus pretiosis.

53. Contrairement à ce qu'affirmait J. B. FRIEDMAN, « Thomas of Cantimpré, De natura rerum : Prologue, Book III and Book XIX », Cahiers d'études médiévales, 2 (1974), p. 107154.

54. Comme il est fait en revanche dans d'autres sections (par exemple avec Experimentator, Orientalis historia...).

55. Outre des vers épars, sont laissés de côté les chap. 30, De gerachite ; 31, De epistite; 34, De peanite ; 38, De exacontalito ; 50, De margaritis vel unione ; 60, De chrisopatio ; Epilogus I, De anulo et gemma, Epilogus II.

56. Dans l'ordre : prologue et liste de Tethel, prologue et liste d'Acatengi/Azareus, liste de Damigeron, bénédiction chrétienne, bénédiction astrologique, noyaux mineurs. 
est donc unique, puisqu'il met en relation de manière toujours différente les divers blocs dans lesquels il ne sélectionne qu'une partie des éléments. Si l'on considère le respect que Thomas porte à ses sources, on peut toutefois soutenir qu'il en reproduit une version partielle, et dont l'ordre est particulier (prologue de Tethel, liste d'Azareus, liste de Tethel, bénédiction chrétienne). Bien qu'un tel témoin n'ait pas été identifié ${ }^{57}$, la liste dite d'Azareus du De lapidibus pretiosis de Thomas a toutefois en commun 23 de ses 24 passages avec la liste du manuscrit Paris, BnF, lat. 6755 ${ }^{58}$. Malgré seulement 14 points communs, la «liste de Tethel» de Thomas présente en revanche d'importantes similitudes ${ }^{59}$ avec celle du manuscrit Paris, BnF, lat. $8454{ }^{60}$. Quant à la formule de bénédiction chrétienne, elle rappelle un passage du Liber subtilitatum de Hildegarde, ce qui donne à penser que ce type de texte a circulé dans le milieu culturel de l'époque ${ }^{61}$. Pour finir, il n'est pas à exclure que le Dominicain ait utilisé un opuscule sur les sceaux en langue

$C f$. D. PINGreE, «The Diffusion of Arabic Magical Texts...»; et K. MeSLER, «The Medieval Lapidary of Tethel/Azareus... ».

57. Une partie seulement des exemples cités par K. MESLER (« The Medieval Lapidary of Tethel/Azareus... », p. 138-140) a été vue, sous forme manuscrite ou imprimée, à savoir : Berlin, Staatsbibliothek Preussischer Kulturbesitz, lat. $2^{\circ} 307$ (956), f. 22r-23r ; Bologna, BU, 101 (135), f. 23r-24r ; Cambridge, TCL, O.2.18 (1122), f. 173v-176r ; Ashb. 1520, f. 5155 ; Leiden, Bibliothek der Rijksuniversiteit, Voss. lat. 4²7, f. 102-109v ; London, BL, Arundel 295, f. 262r-263v, et 342, f. 69r-71r, Cotton Julius D.VIII, f. 121v-123r, Harley 80, f. 105r-106r, Sloane 1784, f. 5v-12v, et 3663, f. 3r-4v ; München, BSB, Clm 18460, f. 80 ; Oxford, BL, Add.A.103, f. 45v-50r, Ashmole 1384, f. 109 et 1471, f. 64v-67v, Digby 79, f. 178v-179v, et 193, f. 28r-30v, Selden Supra 76 (3463), f. 109v-112v ; Paris, BnF, fr. 2009, f. $64 \mathrm{v}$, et lat. 6755 , f. $34 \mathrm{v}-36 \mathrm{v}$; lat. 7156 , f. $186 \mathrm{r}-187 \mathrm{r}$; lat. 7337 , f. 121-129 ; lat. 8454, f. 65v-66r ; lat. 16204, f. 500-507 ; Praha, NK, 2764 (14.H.208) ; Stalden, Collection Jörn Günther ; Wien, ÖNB, 5311, f. 35r-37r.

58. Qui contient 27 passages sur les intailles; 15 d'entre eux partagent l'ordre et le contenu du De gemmarum virtutibus d'Arnold de Saxe ( $c f$. vol. II, p. 75-77). À cause de ses leçons uniques (noms de divinités, mots manquants...), on peut toutefois exclure qu'il s'agisse d'une copie directe des deux. Enfin, il est intéressant de remarquer que le manuscrit est daté d'avant 1267, donc très proche, dans le temps, du LNR (sur cette datation, $c f$. L. DELISLE, Les Manuscrits du comte d'Ashburnham, Paris, 1883, p. 55-60 ; ID., Catalogue des manuscrits des fonds Libri et Barrois, Paris, 1888, p. 216-220 ; C. SAMARAN, R. MARICHAL, Catalogue des manuscrits en écriture latine portant des indications de date, de lieu ou de copiste, Paris, 1962, vol. II, p. 373).

59. Cf. Dlp 70: «Hunc lapidem dicitur Galienus portasse in digito », et De sigillis, f. 66r : « Hoc autem sigillum fertur habuisse Galienus ».

60. Sur ce manuscrit, qui contient 21 passages relatifs à la gravure, voir M. E. HERRERA, «La historia... », p. 158 et 164-165.

61. La même bénédiction se trouve aussi dans trois manuscrits du De floribus d'Arnold de Saxe, à savoir : le ms. Bamberg, Staatsbibliothek, Misc. Nat. 5, ff. 53-54 ; le ms. Erfurt, Wissenschaftliche Allgemeinbibliothek, Ampl. Qu. 368, ff. 81v-82r ; et le ms. Praha, Nàrodni Knihovna, XI.C.2, ff. 250rv. Sur cette question, $c f$. I. DrAELANTS, Un encyclopédiste méconnu du XIII siècle : Arnold de Saxe. Euvres, sources, réception, Louvain-la-Neuve, 2000, p. 454-464, en particulier p. 459 (mise en ligne sur HAL-SHS : http://tel.archivesouvertes.fr/tel-00700745). 
vernaculaire : dans l'incipit du De lapidibus pretiosis, Thomas spécifie en effet «libellum Tethes transtulimus in latinum », et les manuscrits qui transmettent ce passage donnent : « in hoc libello subnotavimus $»^{62}$.

Si l'on analyse les rapports entre De lapidibus pretiosis et le De lapidibus de Marbode, il apparait plausible que le Dominicain utilise une copie de la famille $\delta$-A ou $\delta-B^{63}$. Bien qu'il soit impossible de cerner le sous-groupe utilisé, le livre XIV et ces dernières familles textuelles présentent d'importants points communs (ordre de la matière, interpolations du Damigeron-Evax, anonymat ${ }^{64}$, leçons identiques) ; si l'on considère en outre qu'aucun autre groupe des manuscrits de Marbode $(\alpha, \beta, \gamma, \gamma-\delta, \delta-C$, $\varepsilon)$ n'offre à la fois anonymat et leçons identiques à celles du Liber de natura rerum, et que, dans les manuscrits, les De lapidibus $\delta$-A et $\delta$-B sont souvent suivis par des listes «Tethel-Azareus » et «d'Aaron » ${ }^{65}$, il semble alors assez plausible que le frère se soit servi d'un recueil de ce type ${ }^{66}$.

Si l'on examine à présent les vers du De berillo, ils correspondent à des passages du Bestiaire anglo-normand de Philippe de Thaon (1130$1155)^{67}$. La critique estime que cette dernière œuvre dérive d'un bestiaire

62. Sur cette question, voir L. ThORNDIKE, A History of Magic..., vol. II, p. 391 (et Hildegardis Bingensis Liber subtilitatum... IV, Praefatio, col. 1247) ; J. Evans, P. STUDER, Anglo-Norman lapidaries..., p. 288-296 ; K. MESLER, « The Medieval Lapidary of Tethel/Azareus.... », p. 141-142.

63. Cf. M. E. HERRERA, « La historia... », p. 158, n. 30, et 160, n. 41 ; EAD., Liber..., p. XLV-XLVI.

64. Auteur et œuvre sont cités seulement de manière générique dans Dlp 44 : « secundum vero librum lapidarum»; et Dlp 52 : «auctor libri ». Voir aussi I. DRAELANTS, « La science... », p. 118, n. 2 .

65. Bien que $D l p$ n'omette pas, comme le fait $\delta$, les vers $585-586$, les leçons communes avec $A$ et $B$ se trouvent aux chap. $9 ; 14 ; 15 ; 16 ; 20 ; 21 ; 26 ; 34 ; 48 ; 49 ; 52 ; 60 ; 61$. Il n'est toutefois pas à exclure que Thomas ait utilisé une version indépendante comme celle que renferme le ms. Paris, BnF, Lat. 5009 ; voir à ce sujet M. E. HERRERA, « La historia... », p. 165-166, et EAD., Liber..., p. LXXIX-LXXX.

66. Par exemple dans le ms. déjà cité Paris, BnF, lat. 8454.

67. Dlp 11 : «Berillus, si formam sexangularem habuerit, archus celestis colores efficit in radio Solis, qui, etiam si rotunde forme fuerit velut pomum humectatus aqua, in claritate Solis mortuos carbones vel pannum laneum nigrum vel bullituram, arboris siccam accendit. Unde, quidam egregie satis versificans dixit in persona lapidis berilli : Me dedit insignem virtus que format in ignem / Solis splendorem, non ignis passa calorem. / Sic lux eterna descendit ab arce superna, / Est incarnata, non matre tamen violata »; Bestiaire, v. 30833090 et 3093-3100, p. 111-112 : « Li beriz at vertu en sei./ Le rai del soleil trait a sei. et li raiz est de tel nature / Le beril passe senz frainture ; / E li chalz est de l' altre part, / Que il esprent et bruille et art / Estupes, tundre, drapelez, / Sèches chosetes, estramez ; / [...] Icil beriz nus signefie / Nostre Dame Sainte Marie, / Par le soleil Dé entendum / E par le rai sun fîz pernum ; / Kar si cum li raiz de soleil / A ceste pierë est feeil, / Qu'il entre en li senz uverture / E ultre passe senz frainture »; $c f$. Lapidaire, v. 225-232 et 235-242, p. 212. Sur les rapports entre Bestiaire et Lapidaire, voir C. V. LANGLOIS, La Connaissance de la nature et du monde au Moyen Âge, Paris, 1927, p. 6-10 ; P. MEYER, « Les plus anciens lapidaires français »..., p. 484-488 ; et J. EVANS, P. STUDER, Anglo-Norman lapidaries..., p. 200. 
$B-I s$, et Evans et Studer ajoutent que le Bestiaire et De lapidibus pretiosis ont la même auctoritas latine, qui devrait donc par conséquent être un $B$ $I s^{68}$. Bien que cela ne soit pas à écarter, on peut aussi faire l'hypothèse que Thomas a traduit lui-même le passage concerné du Bestiaire, qui est à ce jour le plus ancien témoin des vers sur le béryl ${ }^{69}$.

Quant aux compléments de ThII, dans le chapitre De pyrophilo Thomas ne renvoie pas aux sources communes sur cette pierre (Physiologus versio $Y$, bestiaires de Guillaume le Clerc et Pierre de Beauvais et PseudoHugo de Folieto ${ }^{70}$ ), mais à la Scriptura Esculapii et au Liber de serpentibus. Or la Scriptura ne correspond ni aux autres «Esculapius » du LNR (De taxo, Medicina ex animalibus $)^{71}$, ni aux textes du même corpus ou dotés d'un titre comparable (De herba vettonica, Herbarius, Ex herbis femininis, Liber Esculapii, Lettres à Alexandre, Azareus, Marcelin, Néron, Octavien, Antiochus, Mecène $)^{72}$. Quant aux excerpta de serpentibus, ils ne dérivent pas de l'ouvrage pseudo-aristotélicien homonyme, mais rappellent plutôt le De mirabilibus auscultationibus ${ }^{73}$. Bien que ces sources restent

68. $C f$. E. Walberg, dans PhILIPPE De ThaOn, Bestiaire ..., p. XXIV-XXVI ; M. F. MANN, Der Physiologus des Philipp von Thaün und seine Quellen, Halle, 1884 ; C. V. LANGLOIS, La Connaissance de la nature..., p. 12-13 ; J. F. MCCUlLOCH, Medieval Latin and French Bestiaries, Chapel Hill, 1962, p. 25-30, 47-54. J. Evans, P. STUDER, Anglo-Norman lapidaries..., p. 203. B-Is a été consulté dans les mss London, BL, Royal 2 C XII, et Oxford, BL, Laud Misc. 247.

69. Cf. C. V. Langlois, La Connaissance de la nature..., p. 3 sq. Des points communs entre Thomas et Philippe sont manifestes aussi dans LNR IV, 54 .

70. Physiologus $Y$ (éd. F. J. CARMOdY, «Physiologus latinus : versio Y»...); Guillaume le Clerc, Bestiaire; Pierre de Beauvais, Bestiaire (version courte: éd. G. R. Mermier, Paris, 1977 ; longue : éd. C. BaKer, Paris, 2010) ; Pseudo-Hugo, De bestiis (éd. J.-P. Migne, PL, vol. 177, col. 134-164 ; voir aussi Bestiaires du Moyen Âge, mis en français moderne et présentés par Gabriel Bianciotto, Paris, 1980 [NdT]. Sur l'identité de ce texte, voir E. KUHRY, « La tradition textuelle du Compendium philosophie », Tabularia, 14 (2014), p. 240, n. 13).

71. Sur ces sources, voir MC II, p. 103-107.

72. Antonii Musae De herba vettonica, Liber Pseudo-Apulei herbarius, Anonymi De taxone liber, Sexti Placiti Liber medicinae ex animalibus (éd. E. HowALD, H. E. SigERIST, Leipzig, 1927) ; PSEUdO-DIOSCORIDES, De herbis feminis (éd. H. F. KÄSTNER, Hermes, 31 (1896), p. 578-636) ; THESSALI MEDICI De virtutibus herbarum ad Claudium vel Neronem (éd. F. Cumont, dans Catalogus codicum astrologorum graecorum, Bruxelles, 1921, p. 253 262) ; Epistola regis Aegypti ad imperatorem Octavianum (éd. R. HALleuX, J. SCHAMP, Les Lapidaires grecs..., p. 231-232) ; ESCULAPII De chronici passionibus (éd. G. KRAUT, dans Experimentarius medicinae, Strasbourg, J. SсHOTT, 1544, p. 1-79); les autres lettres ont été consultées dans les mss London, BL, Harley 978, 1585 et 4986. Sur l'Herbariencorpus, voir E. HowAlD, H. E. SigERIST, dans Antonii Musae De herba vettonica..., p. v-xxiv; G. BAADER, «Die Anfänge der medizinischen Ausbildung in Abenland bis 1100 », dans La scuola nell'Occidente latino dell'Alto Medioevo, Spolète, 1972, vol. II, p. 669-772 ; et P. KITSON, «Lapidary... Part I... », p. 55-60.

73. ARISTOTELeS, Breviloquim de serpente (éd. L.ZETZNER, Theatrum Chemicum, Strasbourg, 1659-1661, vol.V, p. 880-892); ID., De mirabilibus auscultationibus, col. 845b5.165 (éd. G. LiviUs-ARnOLD, Amsterdam, 1978, p. 38) : cf.W. PrinTZ, 
inconnues ${ }^{74}$, il est probable que le passage sur les serpents soit déjà contenu dans la Scriptura, placée à son tour entre De taxo et Medicina ${ }^{75}$ : c'est en effet une habitude de Thomas que d'attribuer au dernier auteur nommé - en l'occurrence, précisément, l' «Esculapius » du De taxo - les écrits anonymes qui le suivent dans un codex ${ }^{76}$. La Scriptura devait donc être une epistola magico-didactique semblable au De $\operatorname{taxo}^{77}$, de lointaine origine grecque, et peut-être passée par un intermédiaire $\operatorname{arabe}^{78}$; il n'est pas impossible non plus qu'il s'agisse du De membris d'Esculape utilisé par Arnold de Saxe, Barthélemy l'Anglais, Vincent de Beauvais et Albert le Grand ${ }^{79}$.

Les ajouts médicaux ou personnels de Th II semblent donc dériver de gloses ou de compendia pratiques ou thérapeutiques au De lapidibus ${ }^{80}$ : presque tous ces ajouts, en effet, sont des interpolations à Marbode, et concernent des pierres courantes dans l'Europe du XIII ${ }^{\mathrm{e}}$ siècle ${ }^{81}$.

«Gilgamesch und Alexander», Zeitschrift der Deutschen Morgenländischen Gesellschaft, 85 (1931), p. 196-206. L'analogie entre l'Epistola du LNR et celle du De venenis de Pietro d'Abano est intéressante : voir L. THORNDIKE, A History of Magic..., vol. II, p. 265-266, et N. WeILL-PAROT, Les «Images astrologiques »..., p. 528.

74. Outre les sources déjà énumérées, on a consulté les textes grecs dans R. HALLEUX, J. SChAMP, Les Lapidaires grecs..., p. 1-189, et F. DE MElY, C. E. Ruelle, Les Lapidaires de l'Antiquité et du Moyen Âge, Paris, 1896-1902, vol. II-III. Ont été également pris en considération: 1. les catalogues des bibliothèques de Berlin, Cambridge, Édimbourg, Heidelberg, Londres, Munich, Oxford, Paris, Prague, Rome, Saint-Gall et Wolfenbüttel ; 2) H. OMOnt, Catalogue général des manuscrits des bibliothèques publiques de France, Paris, 1890 , t. XI ; 3) L. ThORNDIKE, P. KIBRE, A Catalogue of Incipits of Mediaeval Scientific Writings in Latin, Londres, 1963.

75. Voir le ms. London, BL, Harley 978 : entre les deux œuvres s'est glissé un texte médical, De moro (f. 104v-106v). Pour des Epistolae Esculapii différentes mais toujours liées au De taxo, voir le ms. Paris, BnF, Lat. 16195, f. 3v-5r.

76. Cf. V. Rose, «Aristoteles De lapidibus...», p. 337-338 ; L. THORNDIKE, A History of Magic..., vol. I, p. 600, n. 2 ; MC II, p. 51-63, 103-107.

77. Cf. A. DieTERICH, Abraxas, Leipzig, 1891, p. 161-163; W. SPEYER, Die literarische Fälschung im heidnischen und christlichen Altertum, Munich, 1971, p. 80-84. Étant donné la tendance qu'a Thomas à incorporer des gloses, on ne peut exclure que la Scriptura soit une note marginale.

78. Cf. F. SeZgin, Medizin, Pharmazie, Zoologie, Tierheilkunde, Leyde, 1970, vol. III, p. 47, et aussi l'origine clairement grecque du mot pyrophilus.

79. $C f$. L. ThORnDIKe, A History of Magic..., vol. II, p.431-432 et 496, et aussi ARnoldus SAXo, De floribus rerum naturalium (éd. E. StANGe, Die Enzyklopädie des Arnoldus Saxo, 1906, vol. I-III) ; Bartholomaeus Anglicus, De proprietatibus rerum, Strasbourg, 1491 ; AlBertus Magnus, De mineralibus (éd. A. Borgnet, p. 1-116) ; Vincentius Bellovacensis, Bibliotheca Mundi, Douai, 1624.

80. Comme, par exemple, Marbodus Redonensis, Medical Prose Lapidary (éd. J. M. RidDLE, Marbode of Rennes..., p. 122-125).

81. Seule exception dans $D l p 24 ; 40$, où Thomas interpole Solin et Isidore. L'idée dérive du croisement avec Dlp, E. H. BYRNE, « Some Medieval Gem Stones and Relative Values », Speculum, 10 (1935), p. 177-187 ; J. M. RidDle, Marbode of Rennes...; C. LeCOUTEUX, Dictionnaire... ; et W. Schumann, Gemstones of the World, New York, 1977. 
Pour conclure, sur la base de ce qui a été dit concernant la période de composition, les erreurs et les dimensions du Liber de natura rerum, il semble raisonnable de penser non seulement que le religieux s'est aidé d'anthologies lithologiques, mais aussi qu'il a puisé dans de volumineux manuscrits-sources des notes à organiser, compléter et assembler à des moment divers ${ }^{82}$.

Thomas de Cantimpré construit son livre XIV avec soin, mettant en évidence les entrées specialiter, choisissant des sources et des données pratiques proches dans le temps et l'espace, et passant sous silence les références subversives ou ennuyeuses aux mythes, à la magie et à la morale ; ces données sont ensuite mêlées dans des chapitres à plusieurs facettes, concrets, bien argumentés, à l'organisation souvent semblable (l'aspect des pierres, leurs propriétés médico-pratiques, les lieux où on les trouve), et où les sources ne sont pas critiquées mais retaillées avec attention ${ }^{83}$.

Le De lapidibus pretiosis s'avère par conséquent un parfait miroir $\mathrm{du}$ «style encyclopédique » de Thomas de Cantimpré : tout son Liber de natura rerum est de fait construit de manière semblable, et s'impose comme une summa naturaliste et descriptive à l'identité bien définie et unique, qui introduit au monde aussi de manière pragmatique. Ces caractères sont encore accentués dans Th II : avec l'ajout de parties pratiques, cette dernière rédaction élargit encore l'interaction concrète avec la Nature. Le Brabançon réussit ainsi à atteindre ses buts dominicains : en montrant la Nature avec précision et pragmatisme, non seulement il met en valeur la bonté intrinsèque de la Création, mais aussi - en contrepoint - celle du Créateur unique et bon.

82. Voir P. AIKEN, « The Animal History... », p. 217, et MC II, p. 64-65, 123, 129, 141, 148.

83. Ces caractéristiques valurent au $D l p$ une grande popularité : c'est ce que prouvent 1. le nombre de manuscrits ne transmettant que cette section ( $15 \%$ de ceux du $L N R$ ) et 2. la contemporanéité de certains avec Thomas de Cantimpré (par ex., le ms. Wien, ÖNB, 2442). Sur cette question, $c f$. L. THORNDIKE, « De Lapidibus », Ambix, 8 (1960), p. 6-23 ; et H. J. HeRMANN, Die deutschen romanischen Handschriften. Band: Die illuminierten Handschriften und Inkunabeln der Nationalbibliothek in Wien. Teil: Die deutschen romanischen Handschriften, Leipzig, 1926, vol. II, p. 259. 


\section{Annexe}

Cette Annexe est divisée entre titres de chapitres et sources utilisées : elle montre ainsi comment Thomas lie entre elles ses sources (auxquelles il fait référence cidessous pour chaque titre de chapitre). Outre les abréviations et symboles adoptés plus haut, on utilise aussi ici les abréviations suivantes :

\section{Liste des abréviations}

\begin{tabular}{|c|c|}
\hline$[\ldots]$ & ajout de $T h I I$ \\
\hline$(\ldots)$ & glose ou interpolation sur les sources \\
\hline$?$ & incertitude \\
\hline $1 \ldots 1$ & leçon alternative de $T h I I$ \\
\hline n.i. & non inveni \\
\hline Tot. & Total \\
\hline A & Aristote, Meteorologica \\
\hline$A p d^{I / I}$ & Auctoritates pratiques ou descriptives non reconnues de Th I/Th II (n.i.) \\
\hline $\mathrm{Au}$ & saint Augustin, De civitate Dei \\
\hline AN & Alexander Neckam \\
\hline$n r / l d S$ & De naturis rerum/De laudibus divine Sapientie \\
\hline B & Beda Venerabilis, Hexaëmeron \\
\hline$B i$ & Vulgate \\
\hline Ex & Livre de l'Exode \\
\hline Gl & Glossa super Exodum \\
\hline $\mathrm{DE}$ & Damigeron-Evax, De lapidibus \\
\hline$D p$ & Sources De pyrophilos \\
\hline$S E / s$ & Scriptura Esculapii/De serpentibus \\
\hline $\mathrm{HB}$ & Hildegardis Bingensis, Liber subtilitatum diversarum naturarum creaturarum \\
\hline Is & Isidorus Hispalensis, Etymologiae \\
\hline IV & Jacques de Vitry, Orientalis historia \\
\hline$L m^{I I}$ & Lapidaire médical de Th II (n.i.) \\
\hline M & Marbode de Rennes, De lapidibus \\
\hline $\mathrm{P}$ & Pline l'Ancien, Naturalis historia \\
\hline $\mathrm{PC}$ & Petrus Comestor, Historia scholastica \\
\hline PT & Philippe de Thaon, Bestiaire/Lapidaire \\
\hline $\mathrm{Pla}$ & Pseudo-Platearius, De aromaticis speciebus (n.i.) \\
\hline$Q$ & Questiones (n.i.) \\
\hline S & Solin, Polyhistor \\
\hline TA & Tethel-Azareus plus une bénédiction chrétienne \\
\hline $\mathrm{TC}$ & Interventions de Thomas de Cantimpré \\
\hline
\end{tabular}




\section{Les chapitres du De lapidibus pretiosis et leurs sources}

\begin{tabular}{|c|c|c|}
\hline \multicolumn{2}{|c|}{ Cap } & \multirow{2}{*}{$\begin{array}{l}\text { Sources } \\
{[Q] \text { A TC Au AN, } n r \text { M Au TC Au }} \\
\text { PC M TC Au } E x \text { TC }\end{array}$} \\
\hline 1 & Primo generaliter & \\
\hline 2 & De ametisto & $E x$ M Is $A p d^{I}$ \\
\hline 3 & De achate & Ex M \\
\hline 4 & De adamante & TC M (DE) M P M IV M (DE) M \\
\hline 5 & De abeston & M Is \\
\hline 6 & De amianthos & P Is \\
\hline 7 & De allectorio & M \\
\hline 8 & De absintio & $\mathrm{M}\left[\mathrm{Lm}^{I I}\right]$ \\
\hline 9 & De alabandina & TC Is M $\left[\mathrm{Lm}^{I I}\right]$ \\
\hline 10 & De andromanda & M Is \\
\hline 11 & De berillo & $\begin{array}{l}E x \mathrm{M}(\mathrm{DE}) \mathrm{PT} \text { TC PT } A p d^{l} \mathrm{M} \\
\text { (DE) M }\end{array}$ \\
\hline 12 & De borace & $\mathrm{AN}, l d S A p d^{I} \mathrm{AN}, l d S A p d^{I}$ \\
\hline 13 & De carbunculo unus de duodecim & M Ex M Apd \\
\hline 14 & De calcedonio & $\mathrm{M}\left[\mathrm{Lm}^{I I}\right]$ \\
\hline 15 & De corallo & M TC M (DE) M $\left[\mathrm{Lm}^{I I}\right]$ \\
\hline 16 & De crisoprasso & $\mathrm{M}\left[L m^{I I}\right] \mathrm{M}$ \\
\hline 17 & De celidonio lapide & $\mathrm{M}(\mathrm{DE}) \mathrm{M}$ \\
\hline 18 & De calcophano & M \\
\hline 19 & De cristallo & M S M TC M $\left[\mathrm{Lm}^{I I}\right]$ \\
\hline 20 & De crisoleto & $\mathrm{M}\left[\mathrm{Lm}^{I I}\right] \mathrm{M}\left[\mathrm{Lm}^{I I}\right]$ \\
\hline 21 & De ceraunio & M TC \\
\hline 22 & De corneolo & TC M $A p d^{I}$ \\
\hline 23 & De crisoleto & Ex M \\
\hline 24 & De dracontide & $\mathrm{S}\left[\mathrm{Lm}^{I I}\right] \mathrm{S}[\mathrm{P}]$ \\
\hline 25 & De dyonisia & TC Is M \\
\hline 26 & De dyadocho & $\mathrm{M}$ \\
\hline 27 & De amathite & $\mathrm{M}\left[\mathrm{Lm}^{I I}\right] \mathrm{M}$ \\
\hline 28 & De echite lapide & M IV M \\
\hline 29 & De elitropia & M Is M \\
\hline 30 & De elidro lapide & Is $\mathrm{M}\left[\mathrm{Lm}^{I I}\right] \mathrm{M}$ \\
\hline 31 & De granato & $\mathrm{M} \mathrm{Apd} d^{l}[\mathrm{M}]$ \\
\hline 32 & De gagathe & M (DE) M Is \\
\hline 33 & De gelasia & M Is M $\left[L^{I I}\right]$ \\
\hline 34 & De gecolito & TC M \\
\hline 35 & De galaritide & $\mathrm{M}$ \\
\hline 36 & De gagatromeo & M \\
\hline
\end{tabular}




\begin{tabular}{|c|c|c|}
\hline \multicolumn{2}{|l|}{ Cap } & \multirow{2}{*}{$\begin{array}{l}\text { Sources } \\
\text { Is } E x \text { P M P }\left[L^{I I}(\mathrm{DE})\right]\end{array}$} \\
\hline 37 & De iaspide unus de duodecim & \\
\hline 38 & De iacincto & $\mathrm{M} A p d^{l} \mathrm{M}$ \\
\hline 39 & De iudaico & Is \\
\hline 40 & De iscisto & Is $\left[\mathrm{Lm}^{I I}\right]$ \\
\hline 41 & De yride lapide & $\begin{array}{l}\mathrm{M}[\mathrm{M}] \mathrm{M} A p d^{I} \mathrm{M} / \text { nel finale : } \\
A p d^{I I} Q \mathrm{TC} A p d^{I I} /\end{array}$ \\
\hline 42 & De iena & TC M \\
\hline 43 & De liparea lapide & M \\
\hline 44 & De ligurio qui est unus de duodecim & $E x$ M IV P [P] P M (DE) M \\
\hline 45 & De margaritis & M \\
\hline 46 & De magnete & TC M Is \\
\hline 47 & De memphite & Is \\
\hline 48 & De melonite & M \\
\hline 49 & De medo & M \\
\hline 50 & De onichino unus de duodecim & $\begin{array}{l}\text { TC } E x \text { TC } G l \text { Is B TC B TC Pla Is } \\
\text { TC } G l A p d^{l}\end{array}$ \\
\hline 51 & De onice & TC Is M $\left[A p d^{I I}\right] \mathrm{M}$ \\
\hline 52 & De ostolamo & $\mathrm{M}\left[A p d^{I I}\right] \mathrm{M}$ \\
\hline 53 & De orite & $\mathrm{M}$ \\
\hline 54 & De perite vel peridonio & $\mathrm{M}\left[\mathrm{P} L m^{I I}\right] \mathrm{M}$ \\
\hline 55 & De panthera lapide & M TC \\
\hline 56 & De prasio & M Is M Is \\
\hline [57] & De pyrophilos & $D p$ \\
\hline 58 & De saphiro & $E x$ Is M (DE) M (DE) M $A p d^{I}$ \\
\hline 59 & De smaragdo unus de duodecim & TC Ex M \\
\hline 60 & De sardonio & M /al centro: $L m^{I I} \mathrm{M} / \mathrm{M}$ \\
\hline 61 & De sardio unus de duodecim & $E x \mathrm{M}$ TC M $G l$ \\
\hline 62 & De sirio & Is TC \\
\hline 63 & De sarcophago & Is \\
\hline 64 & De samio & Is $\mathrm{P}$ \\
\hline 65 & De succino & Is $\mathrm{P} A p d^{l}$ Is [P] \\
\hline 66 & De speculari & Is $\left[A p d^{I I}\right]$ \\
\hline 67 & De sylonite & M TC M \\
\hline 68 & De sarda & M TC \\
\hline 69 & De topasio unus de duodecim & Ex M (DE) M P \\
\hline
\end{tabular}




\begin{tabular}{|l|l|l|}
\hline \multicolumn{2}{|l|}{ Cap } & Sources \\
\hline 70 & De sculphitos lapidum & TC TA \\
\cline { 2 - 3 } & Hucusque secundum antiquos & TC \\
\cline { 2 - 3 } & Item philosophum Tethes & TA TC \\
\cline { 2 - 3 } & Sculpturis & TA \\
\cline { 2 - 3 } & $\begin{array}{l}\text { Incipit vero quomodo lapides perdunt } \\
\text { virtutes et recuperant naturales }\end{array}$ & TA \\
\cline { 2 - 3 } & $\begin{array}{l}\text { Benedictio super lapides qui consacrandi } \\
\text { sunt }\end{array}$ & TA HB \\
\hline
\end{tabular}

Mattia Cipriani - IRHT-EPHE-LabeEx HASTEC

Un aspect de l'encyclopédisme de Thomas de Cantimpré. La section De
lapidibus pretiosis du Liber de natura rerum

Bien que tous les encyclopédistes du XIII ${ }^{e}$ siècle utilisent un corpus commun de sources, chacun d'eux a une manière personnelle de choisir, "découper " et disposer les contenus tirés de ces auctoritates. Ces modi scribendi attentifs et distinctifs ne reflètent pas seulement les différentes formae mentis et finalités à la base d'une encyclopédie médiévale, mais permettent également à un compilateur (qui recueille les prestigieux matériaux d'autrui) de devenir auteur (qui est prestigieux en soi). Grâce à l'analyse de la structure, des sources et des contenus du De lapidibus pretiosis - le quatorzième livre du Liber de natura rerum, une célèbre encyclopédie écrite entre 1242/1247 et 1260 par le Dominicain flamand Thomas de Cantimpré (1201-1270/1271) -, cet article veut mettre en lumière le "style encyclopédique " exclusif et attentif et les buts précis de son auteur.

De lapidibus pretiosis - Encyclopédisme médiéval - Liber de natura rerum Sources de l'encyclopédisme médiéval - Thomas de Cantimpré

\section{An aspect of Thomas' of Cantimpré Encyclopaedism. The De lapidibus pretiosis Section of the Liber de natura rerum}

Even though all thirteenth century encyclopaedists used a common corpus of sources, each of them had a precise and personal way to choose, "tailorize" and arrange the contents taken from these auctoritates. These peculiar and custom modi scribendi reflect accurately the different formae mentis and purposes behind the encyclopaedic texts, while also permitting the compiler (who collects authoritative materials of others) to become an author (who in turn becomes authoritative). Through the analysis of the structure, contents and sources of De lapidibus pretiosis-the fourteenth book of the widespread encyclopedia Liber the natura rerum (approximately 1242/1247-1260)-, this essay will show the exclusive " encyclopaedic style " and goals of its author, the Flemish Dominican friar Thomas of Cantimpré (1201-1270/1271).

De lapidibus pretiosis - Liber de natura rerum - Medieval encyclopaedism Sources of medieval encyclopaedism - Thomas of Cantimpré 